University of Nebraska - Lincoln

DigitalCommons@University of Nebraska - Lincoln

\title{
Complex magnetic phase in submonolayer Fe stripes on Pt(997)
}

Jan Honolka

Max-Planck-Institut für Festkörperforschung, honolka@fzu.cz

T. Y. Lee

Max-Planck-Institut für Festkörperforschung

K. Kuhnke

Max-Planck-Institut für Festkörperforschung

D. Repetto

Max-Planck-Institut für Festkörperforschung

V. Sessi

Max-Planck-Institut für Festkörperforschung, violetta.sessi@jku.at

See next page for additional authors

Follow this and additional works at: https://digitalcommons.unl.edu/physicsenders

Part of the Physics Commons

Honolka, Jan; Lee, T. Y.; Kuhnke, K.; Repetto, D.; Sessi, V.; Wahl, Peter; Buchsbaum, A.; Varga, P.; Gardonio, S.; Carbone, C.; Krishnakumar, S. R.; Gambardella, P.; Komelj, M.; Singer, R.; Fähnle, M.; Fauth, K.; Schütz, G.; Enders, Axel; and Kern, K., "Complex magnetic phase in submonolayer Fe stripes on Pt(997)" (2009). Axel Enders Publications. 10.

https://digitalcommons.unl.edu/physicsenders/10

This Article is brought to you for free and open access by the Research Papers in Physics and Astronomy at DigitalCommons@University of Nebraska - Lincoln. It has been accepted for inclusion in Axel Enders Publications by an authorized administrator of DigitalCommons@University of Nebraska - Lincoln. 


\section{Authors}

Jan Honolka, T. Y. Lee, K. Kuhnke, D. Repetto, V. Sessi, Peter Wahl, A. Buchsbaum, P. Varga, S. Gardonio, C. Carbone, S. R. Krishnakumar, P. Gambardella, M. Komelj, R. Singer, M. Fähnle, K. Fauth, G. Schütz, Axel

Enders, and K. Kern 


\title{
Complex magnetic phase in submonolayer Fe stripes on $\operatorname{Pt}(997)$
}

\author{
J. Honolka, ${ }^{1, *}$ T. Y. Lee, ${ }^{1}$ K. Kuhnke, ${ }^{1}$ D. Repetto, ${ }^{1}$ V. Sessi, ${ }^{1}$ P. Wahl,${ }^{1}$ A. Buchsbaum,${ }^{2}$ P. Varga,${ }^{2}$ S. Gardonio, ${ }^{3}$ \\ C. Carbone, ${ }^{3}$ S. R. Krishnakumar, ${ }^{4}$ P. Gambardella, ${ }^{5}$ M. Komelj, ${ }^{6}$ R. Singer, ${ }^{7}$ M. Fähnle, ${ }^{7}$ K. Fauth, ${ }^{7}$ G. Schütz, ${ }^{7}$ \\ A. Enders, ${ }^{1}$ and K. Kern ${ }^{1,8}$ \\ ${ }^{1}$ Max-Planck-Institut für Festkörperforschung, Heisenbergstrasse 1, 70569 Stuttgart, Germany \\ ${ }^{2}$ Institut für Allgemeine Physik, Technische Universität Wien, Wiedner Hauptstrasse 8-10/134, A 1040 Wien, Austria \\ ${ }^{3}$ Istituto di Struttura della Materia, Consiglio Nazionale delle Ricerche, Area Science Park, 34012 Trieste, Italy \\ ${ }^{4}$ International Centre for Theoretical Physics (ICTP), Strada Costiera 11, 34100 Trieste, Italy \\ ${ }^{5}$ Institució Catalana de Recerca i Estudis Avançats (ICREA) and Centre d'Investigacions en Nanociència i Nanotecnologia (CSIC-ICN), \\ UAB Campus, 08193 Bellaterra, Spain \\ ${ }^{6}$ Jožef Stefan Institute, Jamova 39, SI-1000 Ljubljana, Slovenia \\ ${ }^{7}$ Max-Planck-Institut für Metallforschung, Heisenbergstrasse 13, 70569 Stuttgart, Germany \\ ${ }^{8}$ Institut de Physique des Nanostructures, École Polytechnique Fédérale de Lausanne, 1015 Lausanne, Switzerland \\ (Received 8 July 2008; revised manuscript received 6 January 2009; published 27 March 2009)
}

\begin{abstract}
Correlations between magnetism and morphology of iron nanostructures of monatomic height on $\operatorname{Pt}(997)$ substrates are studied using X-ray magnetic circular dichroism as well as scanning tunneling microscopy and helium scattering. A drastic collapse of the average magnetization by more than a factor of 4 is observed when increasing the iron coverage from 0.1 to $0.2 \mathrm{ML}$. This effect goes along with a softening of the magnetic anisotropy energy and a gradual reorientation of the magnetic easy axis from in plane to out of plane. The experimental findings together with electronic density-functional calculations suggest the formation of a complex magnetic phase in corrugated rim regions of Fe islands, leading to both ferromagnetic and antiferromagnetic exchange couplings of Fe moments depending on their various local bonding configurations.
\end{abstract}

DOI: 10.1103/PhysRevB.79.104430 PACS number(s): 75.75.+a, 75.50. $-\mathrm{y}, 75.70 .-\mathrm{i}, 75.30 .-\mathrm{m}$

\section{INTRODUCTION}

Low-dimensional magnetic structures on surfaces have been under intense study in recent years. The investigated geometries span the spectrum from single atoms, ${ }^{1-3}$ via monatomic chains ${ }^{4}$ and two-dimensional (2D) islands ${ }^{5,6}$ all the way to three-dimensional (3D) nanoclusters. ${ }^{7,8}$ In these systems the magnetic ground state is usually defined by a complex interplay of symmetry, nearest-neighbor distance, and hybridization with the substrate. Nontrivial magnetic groundstate configurations such as antiferromagnetically coupled and exchange-biased thin-film heterostructures have been under research the last two decades, triggering new powerful applications. In recent years, however, experimental and theoretical works are increasingly interested in tailoring complex magnetic configurations on the atomic scale. The theory of spin frustration and skyrmions are two prominent examples. ${ }^{9,10}$ Recent experiments indeed show that thin $3 d$ metal films on $5 d$ substrates exhibit a variety of complex magnetic pattern on the atomic scale. ${ }^{11,12}$ In this work we report the drastic collapse of the average magnetization by a factor of 4.4 in submonolayer Fe nanostructures on $\mathrm{Pt}(997)$ when increasing the iron coverage from 0.1 to $0.2 \mathrm{ML}$. The experimental results suggest that the drastic effect is due to a complex coordination-dependent competition of ferromagnetic (FM) and antiferromagnetic (AFM) exchange coupling of Fe moments.

\section{EXPERIMENT AND RESULTS}

Fe structures of monatomic height as sketched in Fig. 1(b) were prepared in situ on vicinal $\operatorname{Pt}(997)$ surfaces under ultra- high vacuum conditions. The Pt(997) surface was carefully prepared using standard procedures described in earlier publications. ${ }^{13}$ Using low-energy electron diffraction (LEED) the formation of a regular array of (111)-oriented terraces of about $20 \AA$ width (eight atomic rows with a standard deviation of one row) separated by monatomic steps was verified for every sample, which leads to the characteristic superstructure in the pattern shown in Fig. 1(a) as discussed in Ref. 14. Iron is thereafter evaporated onto $\mathrm{Pt}(997)$ from a rod $(99.99 \%$ purity) by electron bombardment. The $\mathrm{Pt}(997)$ substrate was held at a temperature of $330 \mathrm{~K}$ to allow diffusion of $\mathrm{Fe}$ atoms to the step edges, and thus, to promote step-flow growth. During the growth, the pressure in the chamber is less than $2 \times 10^{-10}$ mbar. The coverage calibration using a microbalance is based on the density of bulk Fe and has to be converted to monolayer (ML) units by considering the actual areal density of the adlayers as discussed elsewhere. ${ }^{14}$ In the following, the nominal coverage $x$ will be given in $\mathrm{ML}$ units according to the atomic density of a pseudomorphic fcc structure on $\mathrm{Pt}(111)$ terraces.

Scanning tunneling microscopy (STM) was used to gain exact information about the quality of the growth with increasing Fe coverages. The images in Fig. 1 are taken at $T$ $=4 \mathrm{~K}$ after sample preparation at room temperature. At $\mathrm{Fe}$ coverages around $x=0.1 \mathrm{ML}$ the $\mathrm{Fe}$ atoms decorate the steps and form well-ordered monowires of atoms along the [1 $\overline{1} 0]$ direction. The high degree of order at this coverage leads to a pronounced peak in thermal energy atomic scattering (TEAS) at grazing incidence as shown in Fig. 1(c) (for a detailed discussion of the TEAS results we want to refer to the work of Lee et al. ${ }^{15}$ ). Beyond monowire coverages, however, the order is lost and no further peaks are observed. 
a)
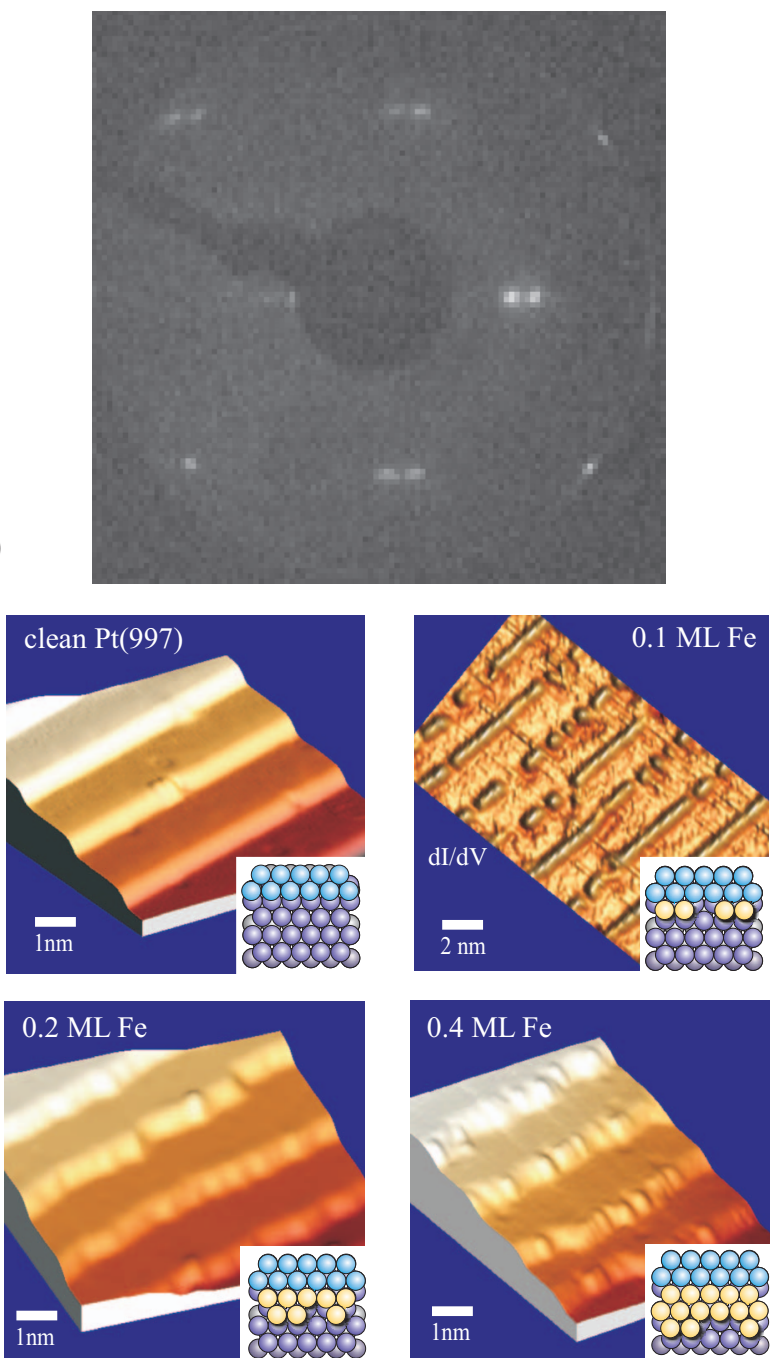

b)
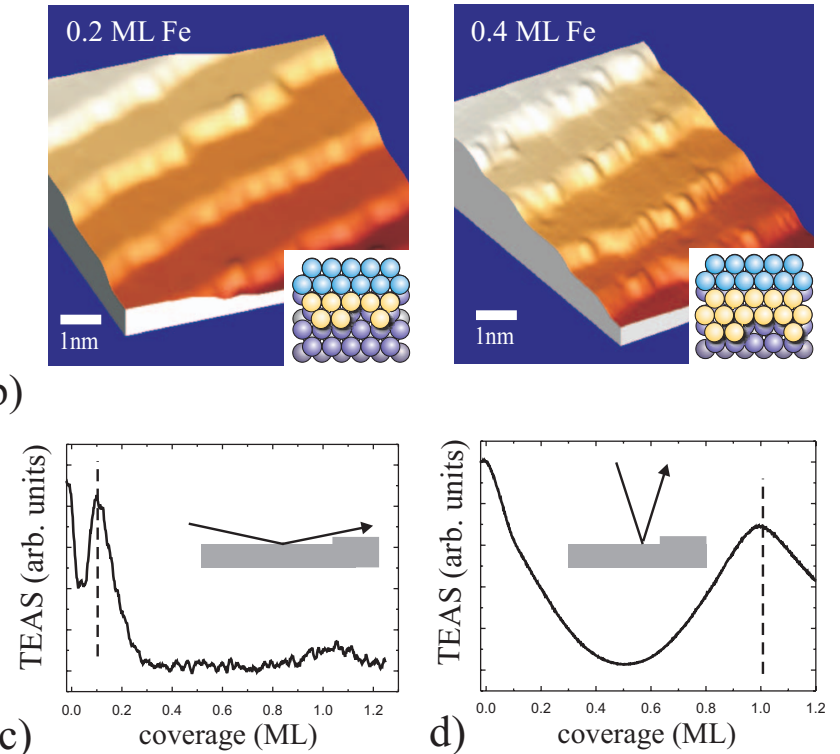

d)

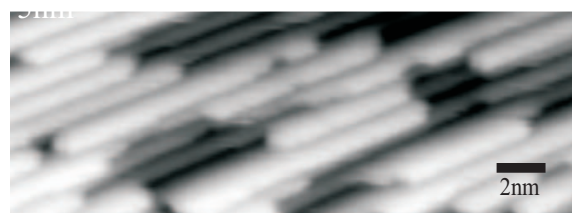

e)

FIG. 1. (Color) Growth behavior of $\mathrm{Fe}$ on $\mathrm{Pt}(997)$ at $T$ $=300$ K. (a) In situ LEED image of a clean Pt(997) substrate taken during the XMCD experiments. (b) Typical STM images for coverages between $0 \leq x \leq 0.4 \mathrm{ML}$. The sketches in the insets model the geometry (Fe shown in yellow). [(c) and (d)] TEAS at grazing and normal incidence measured perpendicular to the substrate steps. (e) Large-scale STM image taken at $x=0.7 \mathrm{ML}$ showing the 2D equivalent to a Stranski-Krastanov growth.

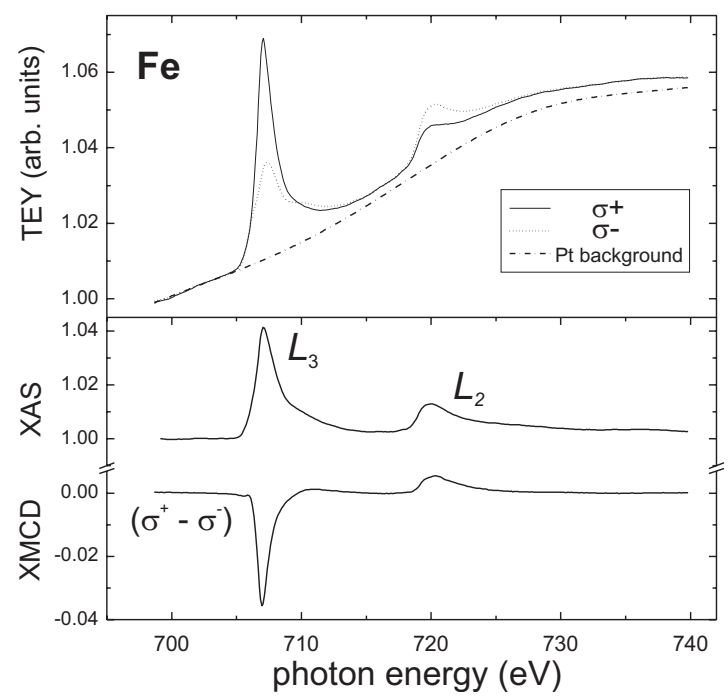

FIG. 2. Upper panel: Fe $L_{3,2}$ edges for $H= \pm 5.5 \mathrm{~T}$ (full and dotted lines) measured in the TEY mode. A background spectrum of the clean Pt substrate is shown as a dashed-dotted line. Lower panel: background corrected XAS of $\mathrm{Fe}$ and respective XMCD signal.

Indeed, the STM images reveal a more and more corrugated step-flow growth with increasing coverages $0.13<x$ $<1$ ML. Moreover, on a larger scale it is evident from STM at around 0.7 ML shown in Fig. 1(e) that beyond monowire coverages, the 2D equivalent to Stranski-Krastanov growth is observed, which leads to the formation of blocks. A careful statistical evaluation of the large scale STM images shows that block formation happens at about $(75 \pm 5) \%$ of the $\mathrm{Pt}$ step edge. While the grazing incidence TEAS signal is sensitive to processes at the step edges, the nongrazing incidence provides information on the terrace morphology. The peak appearing at exactly 1 ML [see Fig. 1(d)] is a proof of the pseudomorphic growth of Fe on $\mathrm{Pt}(997)$ and shows the strong tendency of Fe to fully cover the Pt terraces before forming $\mathrm{Fe}$ double layers.

The magnetic properties of samples with varying Fe coverage were examined extensively using the $\mathrm{x}$-ray magnetic circular dichroism (XMCD) technique. X-ray absorption spectra (XAS) at the Fe $L_{3,2}$ absorption edges (between 690 and $740 \mathrm{eV}$ ) were recorded in the total electron yield (TEY) mode for different angles $\Theta$ between the surface normal and the x-ray beam direction perpendicular to the substrate step direction [110]. Magnetic fields were applied collinear to the beam. Before and after the magnetic characterization the samples were carefully checked for oxygen contaminations using the XAS at the O- $1 s$ absorption edge. The measurements were performed in situ at the ESRF, undulator beamline ID8, at a temperature of $T=8 \pm 1 \mathrm{~K}$ and at the bending magnet beamline BESSY II with a base temperature of $T$ $=12 \pm 1 \mathrm{~K}$. Typical XAS for positive and negative circular polarizations $\left(\sigma^{+}, \sigma^{-}\right)$are shown in Fig. 2 together with the Pt background derived from a measurement on a clean $\mathrm{Pt}(997)$ substrate (dashed-dotted line). Due to the applied field of several tesla a pronounced XMCD signal $\left(\sigma^{+}-\sigma^{-}\right)$is observed, which reflects the spin (orbital) polarization of the 

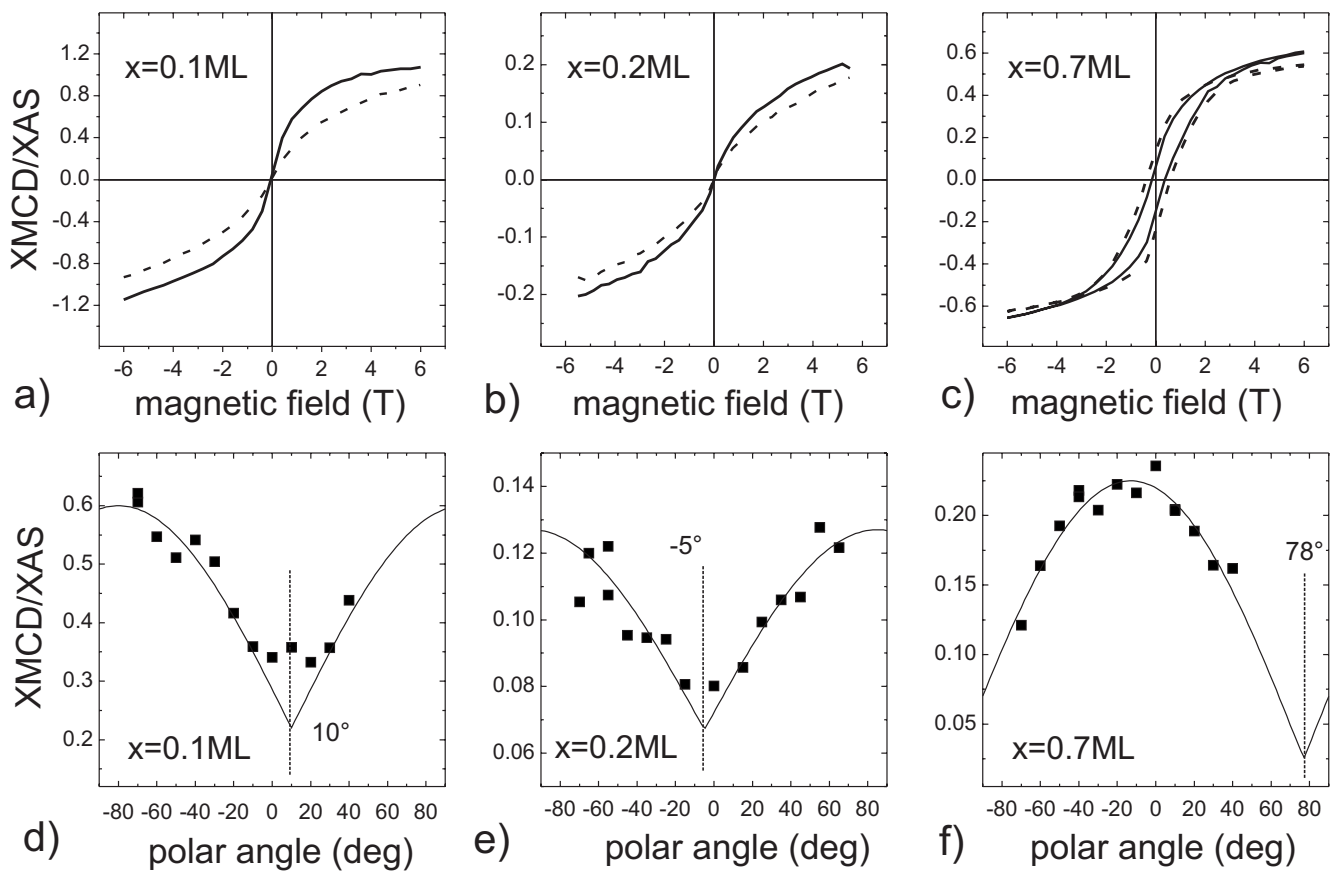
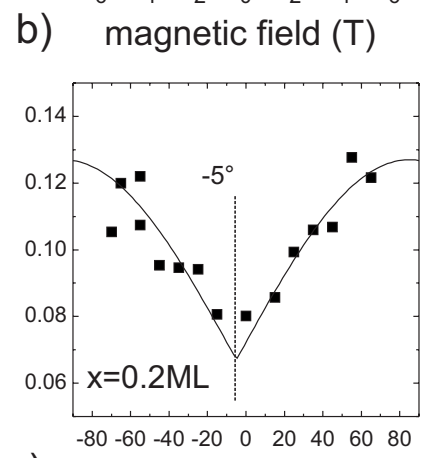

e)

polar angle (deg)
C) magnetic field (T)

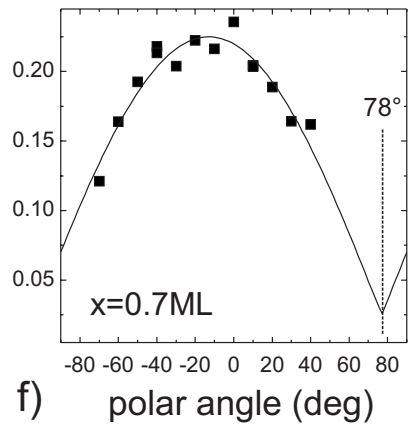

FIG. 3. [(a)-(c)] Polar (dashed lines) and in-plane (full lines) magnetization loops taken at $7 \mathrm{~K}$ for coverages $x=0.1,0.2$, and $0.7 \mathrm{ML}$. Lower panel: magnetization measured [(d)-(e)] in a field of $1 \mathrm{~T}$ applied and (f) in remanence for different directions. The plotted quantity $\mathrm{XMCD}$ /XAS (the peak XMCD intensity normalized to the peak XAS intensity at the $L_{3}$ edge) is a direct measure of the average magnetization per Fe atom along the field direction.

Fe $3 d$ states. As a first approximation the XMCD signal is proportional to the average Fe moments. More precisely, $\mathrm{XMCD} / \mathrm{XAS}=\frac{\left(\sigma^{+}-\sigma^{-}\right)}{\left(\sigma^{+}+\sigma^{-}\right) / 2} \propto \Sigma \cdot M$, that is, the XMCD signal normalized to the nondichroic intensity is proportional to the projection of the total average magnetization $M$ on the vector of the incoming photon spin $\Sigma$. In Figs. 3(a)-3(c) the magnetization curves are shown for coverages $x=0.1,0.2$, and $0.7 \mathrm{ML}$. The measurements were taken at $T=7 \mathrm{~K}$ for polar angles $\Theta=0^{\circ}$ and $\Theta=65^{\circ}$. First we want to point out that both low-coverage samples exhibit superparamagnetic behavior, reflecting their limited dimensionality. Remanence, however, is clearly observed at $x=0.7 \mathrm{ML}$ indicating that at larger coverages, the criteria for superparamagnetic blocking are fulfilled. Measurements at intermediate coverages, not displayed here, demonstrate that the transition from superparamagnetism to blocking happens at coverages around $x$ $=0.4 \mathrm{ML}$. A similar blocking behavior was reported by Cheng et al. ${ }^{16}$ who studied comparable Fe/Pt(997) systems using the magneto-optical Kerr effect (MOKE) technique. The known limited sensitivity of MOKE compared to XMCD confines their magnetic measurements to coverages larger than $x=0.5$ ML. Striking effects, however, occur below this coverage. Figures 3(a) and 3(b) show that the magnitude of the magnetization at highest available fields of 5.5 $\mathrm{T}$ is reduced by about a factor of 4.4 when increasing the coverage from 0.1 to $0.2 \mathrm{ML}$, which evidences a drastic collapse of the average magnetic moment per Fe atom between coverages equivalent to approximately a one-dimensional (1D) monowire (0.13 ML) and 2D biwire configuration $(0.26$ $\mathrm{ML}$ ). Comparing the polar (dashed lines) and in-plane (full lines) results furthermore shows that while the monowire sample exhibits a large asymmetry in the magnetic response, at $x=0.2 \mathrm{ML}$ the difference between in-plane and out-ofplane magnetization curves is significantly reduced. Since the magnetic easy-axis direction changes only moderately, this degeneracy can be attributed to a softening of the average magnetic anisotropy energy. The former becomes clear from Figs. 3(d) and 3(e), where the magnetization in a constant magnetic field of $1 \mathrm{~T}$ was measured for different polar angles $\Theta$. Along the hard axis direction the induced moments should be lowest. In the case of monatomic wires $a b$ initio calculations exist which predict a magnetic hard axis close to the polar direction, ${ }^{17}$ which is in good agreement with our measurements at $x=0.1 \mathrm{ML}$. The results in Figs. 3(d) and 3 (e) indicate only a slight reorientation of the magnetic hard axis direction $\Theta_{\circ}$ from $\Theta_{\circ}=10^{\circ}$ to $\Theta_{\circ}=-5^{\circ}$ when going from coverages of $0.1-0.2 \mathrm{ML}$. The softening of the magnetic anisotropy energy is also reflected in the comparably weak angle dependence of the measured magnetization in Fig. $3(\mathrm{e})$. At higher coverages of $0.7 \mathrm{ML}$ the magnetic easy axis is finally rotated fully out of plane. This is shown in Fig. 3(f), where the remanent magnetization $\Sigma \cdot M$ is plotted for different angles $\Theta$. The angle $\Theta_{\circ}=78^{\circ}$ of the hard axis direction is found close to the in-plane direction, in agreement with earlier SMOKE measurements at higher temperatures $T$ $>130 \mathrm{~K}$, where $1 \mathrm{ML} \mathrm{Fe}$ on $\mathrm{Pt}(997)$ was shown to be out of plane magnetized. ${ }^{14}$ Resuming the results of Fig. 3, the collapse of the average magnetization around $x=0.2 \mathrm{ML}$ is accompanied by a softening of the magnetic anisotropy energy, and at higher coverages a gradual reorientation of the magnetic easy axis from the in-plane to the out-of-plane direction is observed. ${ }^{18}$

The average effective spin $\left(m_{S}^{\text {eff }}=m_{S}+7 m_{T}\right)$ and orbital $\left(m_{L}\right)$ moments per atom and $3 d$ hole $n_{h}$ can be quantified by analyzing XMCD spectra taken at the highest fields using the 


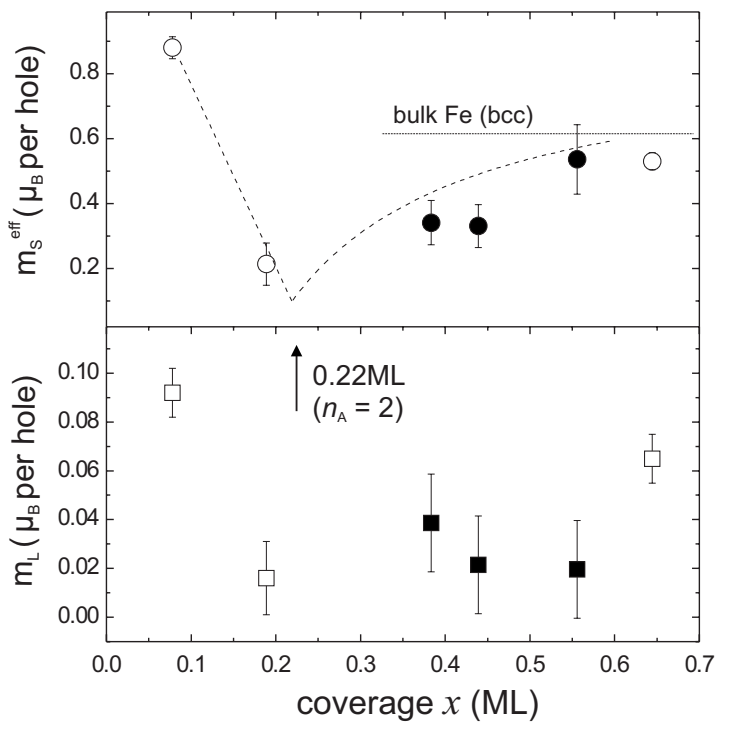

FIG. 4. Average effective spin and orbital moments per atom and $3 d$ band hole measured in the magnetic easy-axis directions at the respective coverages. The measurements were performed at ESRF (open symbols) and BESSY II (full symbols). The dashed line in the upper panel represents a fit of the effective spin moment using Eq. (1).

sum rules for metal $3 d$ elements. ${ }^{19,20} m_{T}$ is the contribution of the magnetic-dipole term. In Fig. 4 the results are shown as a function of the Fe coverage. All values have been measured along the corresponding easy-axis directions derived from Fig. 3. The collapse of the magnetic moments around $x=0.2$ ML is clearly reflected both in the spin and the orbital moments. However, it should be stressed that the moments derived for $x=0.2$ ML can only serve as a lower limit since the shape of the magnetization curve in Fig. 3(b) does not show full saturation at highest available fields. Figure 4 shows that beyond this critical coverage, however, the moments start to recover gradually and at $x=0.7 \mathrm{ML}$ almost reach values measured in bulk Fe. The importance of this critical coverage is further underlined by the observed softening of the magnetic anisotropy at coverages close to $x$ $=0.2 \mathrm{ML}$ as shown in Fig. 3. Theory predicts $n_{h}$ to be between 3.5 and 3.7 and rather stable for differently coordinated $\mathrm{Fe}$ atoms. With $n_{h}=3.5$ we derive an effective spin contribution of $\left(m_{S}+7 m_{T}\right) \approx 3.1 \mu_{B}$ for monatomic wires. From ab initio calculations of monatomic wires at Pt steps using linear muffin-tin orbital (LMTO) and full-potential linearized augmented plane-wave (FLAPW) methods, the contribution of $7 m_{T}$ can be as large as $-0.5 \mu_{B} \cdot{ }^{21}$ For the orbital moments we derive $m_{L} \approx 0.3 \mu_{B}$ which agrees well with the calculations using the LMTO method, while FLAPW overestimates the actual value.

\section{DISCUSSION}

When discussing changes in the magnetic moments one has to keep in mind that XMCD measures the sample average magnetization. Thus, the observed collapse of the magnetic moments at intermediate coverages can in principle be either due to a decrease in the moment of each $\mathrm{Fe}$ atom separately (true intrinsic low-spin phase) or due to AFM coupling and/or canting of $\mathrm{Fe}$ moments with respect to each other. Iron is known to exhibit a complex diagram of magnetic phases depending strongly on the structural environment of the individual $\mathrm{Fe}$ atoms. Both theoretical and experimental studies of bulk $\gamma$-Fe showed the formation of intrinsic FM high- and low-spin as well as AFM phases depending on the lattice constants. We can use our extensive STM and TEAS studies of Fe on Pt(997) to try to correlate morphology and the appearance of small average magnetization in our samples. In the following we will do this using a simple two-phase model.

TEAS and STM have shown that after completion of a Fe monowire along the $\mathrm{Pt}$ step, the growth continues in a $2 \mathrm{D}$ equivalent to Stranski-Krastanov mode. Only parts of the Pt step edge are covered with larger 2D blocks of Fe [see Fig. 1(e)]. We label these regions as phase $\mathrm{A}$ and the remaining regions of longer $\mathrm{Fe} 1 \mathrm{D}$ monowires at the $\mathrm{Pt}$ step edges as phase B. According to the STM images we can use the twophase model with $75 \%$ and $25 \%$ spectral weights attributed to phase A and phase B, respectively, to disentangle the magnetic properties of the two phases. Figure 5 shows the measured total magnetization curve at $x=0.2$ ML together with the contributions of phases A and B according to the model. For phase B we assumed a signal proportional to that measured for monatomic wires [see Fig. 3(a)] exhibiting an inplane magnetic easy-axis direction. As a result the remaining phase A shows almost no magnetic anisotropy at $x$ $=0.2 \mathrm{ML}$ and exhibits a very shallow magnetic response to magnetic fields up to $5.5 \mathrm{~T}$. According to the two-phase model and taking into account the spectral weights at the critical coverage of $0.2 \mathrm{ML}$, the average magnetization per $\mathrm{Fe}$ atom $\propto \frac{\mathrm{XMCD}}{\mathrm{XAS}}$ in phase $\mathrm{A}$ is by a factor of 12 smaller compared to that of phase $\mathrm{B}$. On the other hand the finite slope at the highest fields indicates that phase A is not saturated.

It is obvious to try to correlate the drastic reduction in the average magnetization in phase A to changes in the symmetry when $\mathrm{Fe}$ atoms are added to the monowire geometry. In ideal monowires the next-neighbor $(\mathrm{NN}) \mathrm{Fe}$ coordination $\mathrm{NN}_{\mathrm{Fe}}$ is 2 and every $\mathrm{Fe}$ atom is laterally coordinated to the $\mathrm{Pt}$ step edges as shown in Fig. 1. Adding another row of $\mathrm{Fe}$ atoms brings $\mathrm{NN}_{\mathrm{Fe}}$ to 4 , and most importantly, breaks the symmetry with respect to the Pt coordination; the second row is only coordinated to Pt from below and instead has a lateral coordination to Fe. Thus, it is expected that the magnetic easy-axis direction of the first and second $\mathrm{Fe}$ rows is not collinear, which can lead to quenching of the average anisotropy similar to that shown theoretically and experimentally for Co biwires along $\mathrm{Pt}(664)$ and $\mathrm{Pt}(997)$ steps, ${ }^{22,23}$ respectively. Indeed the observed softening of the average magnetic anisotropy at $x=0.2$ ML Fe compared to monowire coverages $(x=0.1 \mathrm{ML})$ points to drastic reorientations in the magnetic easy-axis directions due to coordination changes. However, unlike in $\mathrm{Co} / \mathrm{Pt}(997)$ systems in the present case the reorientation is accompanied by the drastic reduction in moments. In the following we want to discuss possible explanations for the reduced average moments in phase A. 


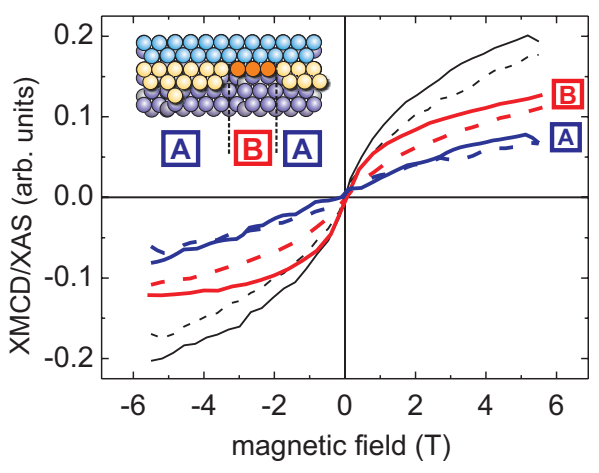

FIG. 5. (Color) Two-phase interpretation of the magnetization curves at $x=0.2$ ML assuming $75 \%$ and $25 \%$ spectral weights attributed to phase A and phase B, respectively. For phase B we assumed a signal proportional to that measured for monatomic wires and the contribution of phase $\mathrm{A}$ is the measured total magnetization curve subtracted by the signal of phase $\mathrm{B}$. The magnetization curves of phase A and phase B are plotted in blue and red for in-plane (full lines) and out-of-plane (dashed lines) geometries. For comparison the total magnetization curve from Fig. 3(b) at 0.2 ML is shown in black color.

\section{A. Intrinsic low-spin phase}

Further analysis of the spectra, not shown here, provides crucial information on this question since drastic changes in the magnetic moments usually leave their traces in the spectral shape. According to Thole and van der $\operatorname{Laan}^{24}$ for a true Fe low-spin phase we expect a decreased branching ratio, $\mathrm{BR}=L_{3} /\left(L_{3}+L_{2}\right)$, in the XAS spectra. The analysis of the peak intensities $L_{3}$ and $L_{2}$, however, show that all samples with $x \leq 0.5 \mathrm{ML}$ exhibit a constant branching ratio of $\mathrm{BR}$ $=(0.67 \pm 0.02)$, equal to the statistical value of $2 / 3$ consistent with the $2 p_{3 / 2}$ and $2 p_{1 / 2}$ core state degeneracies. At higher coverages BR slightly increases. This strongly suggests that the observed collapse of both the average spin and orbital moment is not due to an intrinsic low-spin phase. Moreover, this result also implies that the softening of the magnetic anisotropy at around $x=0.2 \mathrm{ML}$ cannot be explained by the simple fact that the anisotropy energy scales with the intrinsic spin magnetic moment. Therefore, the origin of the decreased anisotropy has to be either a quenching of the directional dependence of the Fe orbital moments in each Fe atom separately ${ }^{25}$ or a wide distribution of on-site easy-axis directions within the sample. Due to the highly anisotropic $\mathrm{Fe}$ bonding configuration at low coverages, we tend to assign the effect to strong variations in the on-site Fe anisotropy.

\section{B. Antiferromagnetic coupling between two Fe wires at the Pt step edge}

At coverages $x=0.2 \mathrm{ML}$ phase A is expected to partially form a second row of $\mathrm{Fe}$ next to the initial monowire (see inset of Fig. 5). One explanation for the drastic reduction therefore is an AFM coupling of the second row of Fe and the monowire. In order to test this heuristic model we performed $a b$ initio density-functional theory (DFT) calculations to model Fe biwires on a $\operatorname{Pt}(997)$ vicinal surface with a supercell of 21 atoms. ${ }^{26}$ The structure was initially assumed

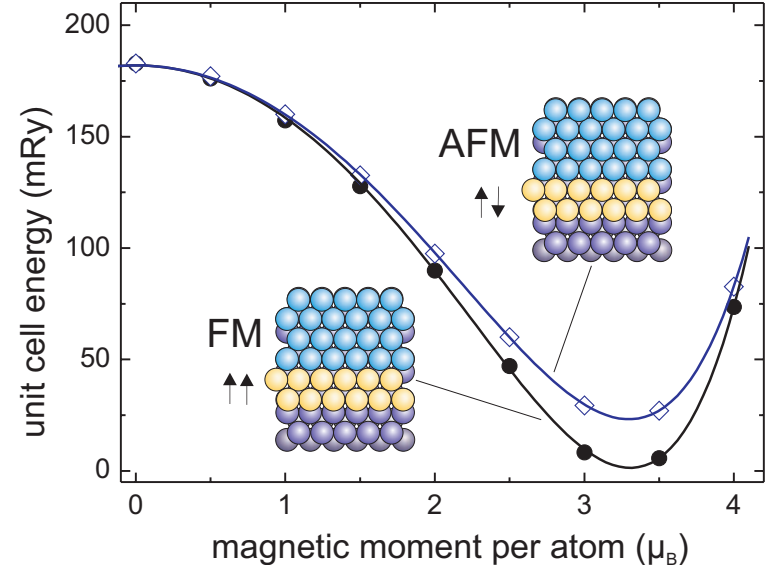

FIG. 6. (Color) DFT calculations to model Fe biwires on a $\operatorname{Pt}(977)$ vicinal surface with a supercell of 18 atoms (Ref. 26). The structure was initially assumed pseudomorphic with next-neighbor distances matching the experimental value for fcc Pt $(2.77 \AA)$. The results of the calculation show that the FM state in ideal biwires is always energetically favorable compared to the AFM state.

pseudomorphic with next-neighbor distances matching the experimental value for fcc Pt $(2.77 \AA)$. The tight-binding linear muffin-tin orbital method (TB-LMTO) (Ref. 27) in atomic-sphere approximation (ASA) was used for the calculation of the dependence of the total energy on the magnitude of the magnetic moments in FM and AFM alignments. These energies were calculated by means of self-consistently determined Lagrange fields. ${ }^{28}$ In contrast to the heuristic model, the results of the calculation in Fig. 6 show that the FM state is always energetically favorable compared to the AFM state. Moreover, the calculations generally show that small Fe moments are energetically unfavored, supporting our interpretation against the formation of an intrinsic low-spin phase. Considering the variation in the next-neighbor distance, the distance between the two wires (i.e., the Fe-Fe distance) and the distance of the Fe-containing layer from the nearest Ptonly layer, we applied the FLAPW method ${ }^{29}$ implemented as the WIEN97 code $^{30}$ in the local spin-density approximation (LDSA). ${ }^{31}$ In all calculations the FM state proves to be stable against structural changes. Full structural relaxation along the perpendicular direction by applying the ABINIT (Ref. 32) code further confirmed the stability of the FM state. In short, from the extensive $a b$ initio DFT calculations we can exclude simple AFM coupling between infinitely long pseudomorphic biwires at the Pt step edges.

\section{Complex noncollinear magnetic structures}

Complex noncollinear magnetic structures usually occur when there is a competition of either FM and AFM exchange or exchange coupling with diverse on-site magnetic anisotropies. The complexity in the magnetism observed in $\mathrm{Fe} /$ $\mathrm{Pt}(997)$ is very likely to stem from the complex structure observed in the STM studies, where (a) the blocks of phase A have a finite width [Fig. 1(e)] and (b) the rim of a block is corrugated on the atomic scale [Fig. 1(b)]. This leads to a manifold of coordination states of $\mathrm{Fe}$ with varying $\mathrm{NN}_{\mathrm{Fe}}$, and 
thus a variation in magnetic properties from atom to atom can be expected. Since in Fe structures exchange coupling constants are usually dominant, we can take the softening of the magnetic anisotropy at the critical coverage of $x$ $=0.2 \mathrm{ML}$ as a strong indication for the presence of local variations in on-site $\mathrm{Fe}$ anisotropies. The reduction in the anisotropy can in this case be explained by a simple random anisotropy model (RAM) as proposed by Herzer. ${ }^{33}$ According to this model the dominating exchange interaction forces the local moments to be collinear, however, due to the randomness in the easy-axis directions the anisotropy averages to small values. In the case of $\mathrm{Fe} / \mathrm{Pt}(997)$ we propose the presence of both FM and AFM couplings depending on the local bonding configuration leading to a small average magnetization and reduced anisotropies according to the RAM. Indeed, FM and AFM exchange couplings in free-standing 2D Fe triangles were predicted by theory depending on their electronic interaction. ${ }^{34}$ Although the influence of the substrate is known to play an important role, the theory supports the notion of the importance of low coordinated rim atoms in corrugated Fe blocks. We also want to mention the possibility of local strain relaxation in the rim region leading to changes in the Fe-Fe distances and interactions.

The above discussion of possible mechanisms behind the formation of the low magnetic phase points to a complex magnetic phase in corrugated rim regions, leading to both FM and AFM couplings of Fe moments depending on the local bonding configurations. We finally want to show that also the observed recovery of the magnetic moment at higher $\mathrm{Fe}$ coverages is compatible with this interpretation. If we assume that due to corrugation effects only the two outmost rim shells of phase A exhibit complex magnetism with vanishing average moments, we can approximate the recovery of the measured total average spin moment as

$$
m_{S}^{\text {eff }} / n_{h}=0.88 \mu_{B}\left[\frac{0.25}{\bar{n}}+\frac{0.75 n_{A}}{\bar{n}} \frac{\left(n_{A}-2\right)}{n_{A}}\right], \quad n_{A} \geq 2,
$$

where $n_{A}$ is the coverage-dependent average number of atomic rows within phase $\mathrm{A}$ (for the monowire phase $n_{B}=1$ by definition), $\bar{n}$ is the average number of atomic rows for both phases, and $0.88 \mu_{B}$ is the measured effective spin moment per atom and $3 d$ hole in the monowires. The factors 0.75 and 0.25 represent the estimation of the spectral weights for phases A and B, according to the STM studies, respectively. Using the calibration of $1 / 8 \mathrm{ML}$ per full atomic row, Eq. (1) represents the trend in the data in Fig. 4 rather well (see dashed line in the range $n_{A} \geq 2$ ). For $1 \leq n_{A} \leq 2$ a linear decrease in the moment is assumed as a guide for the eyes. At higher coverages the model slightly overestimates the data. This could be due to the fact that we assume the average effective spin moment in each $\mathrm{Fe}$ row to be equal to that of the monowire, while it is known that the spin moment usually decreases with increasing $\mathrm{Fe}-\mathrm{Fe}$ coordination.

\section{CONCLUSIONS}

We have studied the low-temperature magnetic behavior of iron nanostructures grown on $\mathrm{Pt}(997)$. A drastic collapse of the average magnetization by a factor of more than 4 is observed when the iron coverage is increased from 0.1 ML to around 0.2 ML. The effect is accompanied by a softening of the magnetic anisotropy energy and a gradual reorientation of the magnetic easy-axis direction from in plane toward out of plane. Using the structural information obtained from extensive surface scanning tunneling microscopy and helium scattering experiments the observed complex magnetism is successfully described within the framework of a two-phase model. One phase is identified as monatomic rows of iron along the step edges of $\mathrm{Pt}$, showing a high average spin magnetic moment per atom of $\left(m_{S}+7 m_{T}\right) \approx 3.1 \mu_{B}$. The second phase consists of blocks of $\mathrm{Fe}$ formed during highly corrugated growth, a 2D equivalent to Stranski-Krastanov growth mode, and shows a considerably reduced magnetization. We ascribe the low magnetization phase to a complex magnetic configuration in corrugated rim regions of $\mathrm{Fe}$ islands, leading to both FM and AFM couplings of Fe moments depending on their various local bonding configurations. The result of this work underlines the complexity of the magnetism in low-dimensional Fe metal structures on surfaces. In contrast to systems such as well-ordered full monolayers of $\mathrm{Fe}$ on $\mathrm{W}(001)$ (Refs. 12 and 35) or $\operatorname{Ir}(111)$ (Ref. 11) where joint $\mathrm{Fe}-\mathrm{Fe}$ and Fe-substrate interactions were shown to promote a long-range-ordered AFM phase, our work addresses the formation of complex Fe magnetism on a local scale in laterally confined nanostructures.

\section{ACKNOWLEDGMENTS}

We would like to thank the synchrotron facilities ESRF and BESSY for their support during the XMCD investigations and the Deutsche Forschungsgemeinschaft (DFG) for financial support within SPP 1153. Partial financial support was also received through the EUROCORES 05-SONS-FP009 SANMAG project of the European Science Foundation.

\footnotetext{
*j.honolka@fkf.mpg.de

${ }^{1}$ P. Gambardella, S. Rusponi, M. Veronese, S. S. Dhesi, C. Grazioli, A. Dallmeyer, I. Cabria, R. Zeller, P. H. Dederichs, K. Kern, C. Carbone, and H. Brune, Science 300, 1130 (2003).

${ }^{2}$ P. Wahl, L. Diekhöner, M. A. Schneider, L. Vitali, G. Wittich, and K. Kern, Phys. Rev. Lett. 93, 176603 (2004).
}

\footnotetext{
${ }^{3}$ V. Madhavan, W. Chen, T. Jamneala, M. F. Crommie, and N. S. Wingreen, Science 280, 567 (1998).

${ }^{4}$ P. Gambardella, A. Dallmeyer, K. Maiti, M. C. Malagoli, W. Eberhardt, K. Kern, and C. Carbone, Nature (London) 416, 301 (2002).

${ }^{5}$ S. Rusponi, T. Cren, N. Weiss, M. Epple, P. Baluschek, L.
} 
Claude, and H. Brune, Nature Mater. 2, 546 (2003).

${ }^{6}$ M. Pratzer, H. J. Elmers, M. Bode, O. Pietzsch, A. Kubetzka, and R. Wiesendanger, Phys. Rev. Lett. 87, 127201 (2001).

${ }^{7}$ D. J. Sellmyer, Nature (London) 420, 374 (2002).

${ }^{8}$ F. Parent, J. Tuaillon, L. B. Stern, V. Dupuis, B. Prevel, A. Perez, P. Melinon, G. Guiraud, R. Morel, A. Barthélémy, and A. Fert, Phys. Rev. B 55, 3683 (1997).

${ }^{9}$ T. E. Saunders and J. T. Chalker, Phys. Rev. Lett. 98, 157201 (2007).

${ }^{10}$ S. Sharma, J. K. Dewhurst, C. Ambrosch-Draxl, S. Kurth, N. Helbig, S. Pittalis, S. Shallcross, L. Nordström, and E. K. U. Gross, Phys. Rev. Lett. 98, 196405 (2007).

${ }^{11}$ K. von Bergmann, S. Heinze, M. Bode, E. Y. Vedmedenko, G. Bihlmayer, S. Blügel, and R. Wiesendanger, Phys. Rev. Lett. 96, 167203 (2006).

${ }^{12}$ A. Kubetzka, P. Ferriani, M. Bode, S. Heinze, G. Bihlmayer, K. von Bergmann, O. Pietzsch, S. Blügel, and R. Wiesendanger, Phys. Rev. Lett. 94, 087204 (2005).

${ }^{13}$ K. Kuhnke and K. Kern, J. Phys.: Condens. Matter 15, S3311 (2003).

${ }^{14}$ D. Repetto, T. Y. Lee, S. Rusponi, J. Honolka, K. Kuhnke, V. Sessi, U. Starke, H. Brune, P. Gambardella, C. Carbone, A. Enders, and K. Kern, Phys. Rev. B 74, 054408 (2006).

${ }^{15}$ T. Y. Lee, S. Sarbach, K. Kuhnke, and K. Kern, Surf. Sci. 600, 3266 (2006).

${ }^{16}$ Ruihua Cheng, K. Yu. Guslienko, F. Y. Fradin, J. E. Pearson, H. F. Ding, Dongqi Li, and S. D. Bader, Phys. Rev. B 72, 014409 (2005).

${ }^{17}$ M. Komelj, D. Steiauf, and M. Fähnle, Phys. Rev. B 73, 134428 (2006).

${ }^{18}$ Further XMCD data, not shown here, prove that in the case of monowires, the in-plane $\left(70^{\circ}\right)$ direction along the steps is slightly harder than the polar direction. At $0.5 \mathrm{ML}$ in-plane measurements along and perpendicular to the steps (both measured at $70^{\circ}$ ) have a similar $M(B)$.

${ }^{19}$ B. T. Thole, P. Carra, F. Sette, and G. van der Laan, Phys. Rev. Lett. 68, 1943 (1992).
${ }^{20}$ P. Carra, B. T. Thole, M. Altarelli, and X. Wang, Phys. Rev. Lett. 70, 694 (1993).

${ }^{21}$ C. Ederer, M. Komelj, and M. Fähnle, Phys. Rev. B 68, 052402 (2003).

${ }^{22}$ G. Bihlmayer, P. Ferriani, St. Baud, M. Ležaić, St. Heinze, and S. Blügel, Ultra-Thin Magnetic Films and Magnetic Nanostructures on Surfaces, in NIC Series Vol. 32: NIC Symposium 2006, edited by G. Münster, D. Wolf, and M. Kremer (John von Neumann Institute for Computing, Jülich, 2006), pp. 151-158.

${ }^{23}$ P. Gambardella, A. Dallmeyer, K. Maiti, M. C. Malagoli, S. Rusponi, P. Ohresser, W. Eberhardt, C. Carbone, and K. Kern, Phys. Rev. Lett. 93, 077203 (2004).

${ }^{24}$ B. T. Thole and G. van der Laan, Phys. Rev. B 38, 3158 (1988).

${ }^{25}$ P. Bruno, Phys. Rev. B 39, 865 (1989).

${ }^{26}$ The supercell is constructed of three layers which represent the fcc (111) stacking and of one additional layer which represents vacuum. The first two layers are filled by eight atoms each. The third layer is filled by two $\mathrm{Pt}$ and three $\mathrm{Fe}$ atoms, whereas the three remaining sites are empty. Each of the two Fe atoms represents one of the two parallel wires.

${ }^{27}$ O. K. Andersen and O. Jepsen, Phys. Rev. Lett. 53, 2571 (1984).

${ }^{28}$ P. H. Dederichs, S. Blügel, R. Zeller, and H. Akai, Phys. Rev. Lett. 53, 2512 (1984).

${ }^{29}$ E. Wimmer, H. Krakauer, M. Weinert, and A. J. Freeman, Phys. Rev. B 24, 864 (1981).

${ }^{30}$ P. Blaha, K. Schwarz, P. Sorantin, and S. B. Trickey, Comput. Phys. Commun. 59, 399 (1990).

${ }^{31}$ J. P. Perdew and Y. Wang, Phys. Rev. B 45, 13244 (1992).

${ }^{32}$ X. Gonze, J. M. Beuken, R. Caracas, F. Detraux, M. Fuchs, G. M. Rignanese, L. Sindic, M. Verstraete, G. Zerah, F. Jollet, M. Torrent, A. Roy, M. Mikami, Ph. Ghosez, J. Y. Raty, and D. C. Allan, Comput. Mater. Sci. 25, 478 (2002).

${ }^{33}$ G. Herzer, IEEE Trans. Magn. 25, 3327 (1989).

${ }^{34}$ S. Uzdin, V. Uzdin, and C. Demangeat, Europhys. Lett. 47, 556 (1999).

${ }^{35}$ L. M. Sandratskii, E. Şaşioğlu, and P. Bruno, Phys. Rev. B 73, 014430 (2006) 